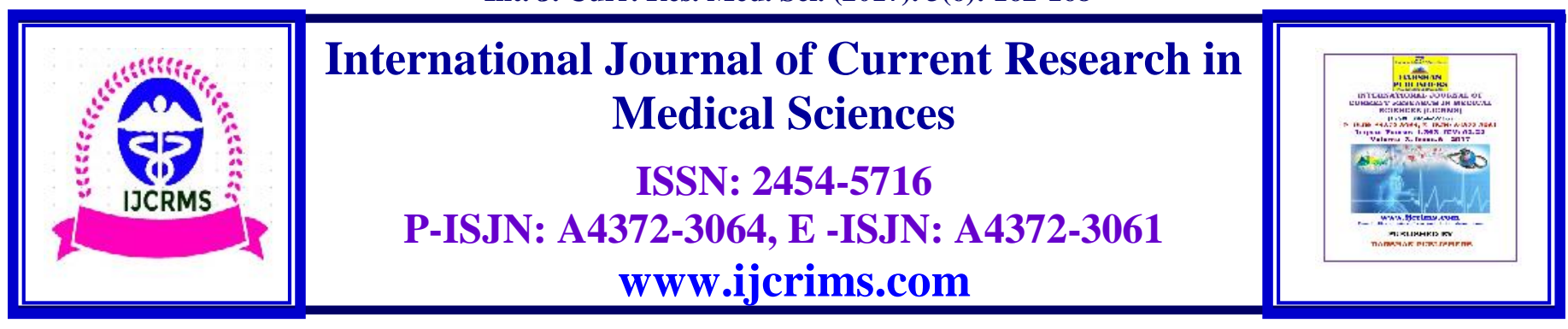

Review Article

Volume 3, Issue 6 -2017

DOI: http://dx.doi.org/10.22192/ijcrms.2017.03.06.021

\title{
Meditation: Techniques and Benefits
}

\author{
Dr. Abdul Wahab Pathath \\ Assistant Professor, Department of Clinical Neurosciences \\ College of Medicine, King Faisal University, Al Ahsa, Saudi Arabia. \\ *Corresponding author: wahabpathath@ gmail.com
}

\begin{abstract}
Meditation is a technique which enables us to relax our body and our mind and, besides, to free our mind of unnecessary thoughts and brain activity. For us Meditating is not an end in itself or a special experience, but just a very efficient technique, which helps us to control our mind and to relax our body. Here discussing different types of meditation techniques and its benefits.
\end{abstract}

Keywords: Meditation, Types of meditation, Benefits of meditation.

\section{Introduction}

Meditation is popularized as a practicing technique for centuries. The term meditation refers to "a family of mental exercises that generally involve calmly limiting thought and attention". Using attentional mechanisms as the basis for the definition, Shapiro (1982) defines meditation as "a family of techniques, which have in common a conscious attempt to focus attention in a no analytical way and an attempt not to dwell on discursive, ruminating thought." Such exercises vary widely and can involve sitting still and counting breaths, attending to a repeated thought, or focusing on virtually any simple external or internal stimulus.

\section{Techniques of meditation}

Meditation is one of the Five Principles of Yoga. It is the practice by which there is constant observation of the mind. It requires a person to focus his mind at one point and stilling the mind in order to perceive the self. Through the practice of Meditation, we will achieve a greater sense of purpose and strength of will. It also helps us achieve a clearer mind, improve our concentration, and discover the wisdom and tranquility within ourself. Meditation is also one of the Eight Limbs of Yoga which leads to Samadhi or Enlightenment. Research shows that the practice of Meditation contributes to our physical and psychological well-being. It can reduce Blood Pressure and relieve stress and pain. Meditating also brings our mind to a level of consciousness that promotes healing or what is known as the alpha state. Achieving the alpha state can help decrease anxiety, depression and other mental, psychological, or emotional problems. Thus meditation process is good to induce relaxation response. 


\section{Transcendental Meditation}

It is one the techniques of meditation which was propagated by Maharshi Mahesh Yogi. The transcendental meditation taught by a teacher, and involves "turning the attention inward towards the subtler levels of thought, until the individual transcends the experience of the subtlest state of the thought and arrives at the source of the thought. This expands the conscious mind and at the same time brings it in contact with the creative intelligence that gives rise to every thought" (Seeman, Nidich, \& Banta, 1972). During the TM the practitioner sits comfortably with eye closed, is given practice and individualized instructions, and practices a thought at progressively earlier and more satisfying steps in its development. Eventually, the practitioner experiences a state of complete mental calmness in which there is no thought but the consciousness is maintained.

\section{Vipassana Meditation}

Vipassana meditation is one of India's most ancient meditative techniques, long lost to humanity, it was rediscovered by Gautama Buddha 2500 years ago (Goenka, 2001). Vipassana meditation has its origin in Theravada and Mahayana Buddhism. Vipassana in Pali means insight. To see things as they really are, in their true perspective, in their true nature. The word Vipassana is combination of two words $\mathrm{Vi}$ and Passana .Vi means "in a special way" and Passa means to see, to observe. HenceVipssana means, "observing in a special way". There are four other concepts relevant to Vipassana namely Sati (mindfulness), Samadhi (absorption), Panna or wisdom, and Bhavana (meditation including Sati, Samadhi and Panna). Vipassana meditation is also known as insight or awareness or mindfulness meditation. Mindfulness is the English translation of Pali word Sati and synonymous to being conscious or aware, taking heed, taking note of, observing, and paying attention.

\section{Yoga Meditation}

This technique may be performed by assuming various postures during various exercises and emphasize the maintenance of calm mind in daily living (Brownstein \&Dembert, 1989). During yoga meditation, the practitioner sits with eye closed, engages in breathing exercises, and concentrates on a word, picture or sound.

\section{Relaxation response}

Benson has proposed a non-religious technique of meditation which he called the "Relaxation Response". The relaxation response as he called it, is a low arousal hypo-metabolic state which can be produced by a variety of techniques. Physiologically it is described as an integrated hypothalamic response with parasympathetic dominance and decreased sympathetic activity. In Benson's method, the meditator, after some muscular relaxation exercises, sits in a quite environment and passively concentrates on his breathing, counting 'one' each time he exhales. When distracting thoughts come up the meditator is asked to ignore them and count 'one' coordinating with outer breath, (Beary and Benson, 1974;Benson, Beary, \& Carol, 1974).

\section{Clinically Standardized Meditation (CSM)}

Carrington (1977) devised her own type of "Mantra" meditation that could be used in clinical practice and research. In CSM the choice of the mantra is left to the individual who chooses one among the sixteen mantras in Sanskrit that Carrington collected. It is even possible for the meditator to concoct his/her own mantra by following some simple rules.

The following instructions illustrates the CSM practice "Having selected your mantra, sit-down comfortably with eyes open resting upon some pleasant object such as a plant, say the mantra out loud to yourself, repeating it slowly rhythmically. Enjoy saying your mantra. Experiment with the sound. Play with it. Let it rock you gently with its rhythm. As you repeat it, say it softer and softer, until finally you let it become almost a whisper".

"Now stop saying mantra out loud, close your eyes, and simply listen to the mantra in your mind. Think it, but do not say it. Let your facial muscles relax, do not pronounce the word, just 
quietly "hear" the mantra, as, for example "Ahnam"..."Ah-nam"..."Ah-nam"...That is all there is to meditating just sitting peacefully, hear the mantra in your mind, allowing it to changes any way it wants - to get louder or softer - to disappear or return - to stretch out or speed up....Meditation is like drifting on a stream in a boat without oars - because you need no oars you are not going any where", (Carrington, 1977, pp. 79-80).

\section{Mindfulness Meditation}

In mindfulness, we observe inward, watching our thoughts without attachment to them. The practice is quite simple. To begin, set your timer or stopwatch for 5 minutes. Then sit in a comfortable position, close your eyes, and focus on your breath. FEEL the breath coming and going, going and coming, through your nose. Your breath becomes the vehicle to carry you towards peace. Now notice how easily you become distracted from the feel of your breath. A thought travels through your mind. That thought leads to another, and another. Finally, you remember that you are supposed to be feeling your breath, and you return. But from where did you return? Where does the mind go? Experiment again and this time you feel a pressure or pain in your body. You follow that pain and another series of thoughts results. And again, you return to the breath. Each time you return to the sensation of your own breath on your nose you have gained a little more control over your own mind.

\section{Simple Meditation}

(1) Choose a quiet spot where you will not be disturbed by other people or by the telephone.

(2) Sit quietly in a comfortable position.

(3) Eliminate distractions and interruptions during the period you will be meditating.

(4) Commit yourself to a specific length of time and try to stick to it.

(5) Pick a focus word or short phrase that's firmly rooted in your personal belief system. A nonreligious person might choose a neutral word like one, peace, or love. Others might use the opening words of a favorite prayer from their religion such as 'Hail Mary full of Grace', "I surrender all to you", "Hallelujah", "Om", etc.

(6) Close your eyes. This makes it easy to concentrate.

(7) Relax your muscles sequentially from head to feet. This helps to break the connection between stressful thoughts and a tense body. Starting with your forehead, become aware of tension as you breathe in. Let go of any obvious tension as you breathe out. Go through the rest of your body in this way, proceeding down through your eyes, jaws, neck, shoulders, arms, hands, chest, upper back, middle back and midriff, lower back, belly, pelvis, buttocks, thighs, calves, and feet.

(8) Breathe slowly and naturally, repeating your focus word or phrase silently as you exhale.

(9) Assume a passive attitude. Don't worry about how well you're doing. When other thoughts come to mind, simply say, "Oh, well," and gently return to the repetition.

(10) Continue for 10 to 20 minutes. You may open your eyes to check the time, but do not use an alarm. After you finish: Sit quietly for a minute or so, at first with your eyes closed and later with your eyes open. Do not stand for one or two minutes.

\section{Walking Meditation}

According to Jon Kabat-Zinn, Director of the Stress Reduction Clinic at the University of Massachusetts Medical Center, one simple way to bring awareness into your life is through walking meditation. "This brings your attention to the actual experience of walking as you are doing it, focusing on the sensations in your feet and legs, feeling your whole body moving, " Dr. KabatZinn explains. "You can also integrate awareness of your breathing with the experience." To do this exercise, focus the attention on each foot as it contacts the ground. When the mind wanders away from the feet or legs, or the feeling of the body walking, refocus your attention. To deepen your concentration, don't look around, but keep your gaze in front of you. "One thing that you find out when you have been practicing mindfulness for a while is that nothing is quite as simple as it appears," says Dr. Kabat-Zinn. "This is as true for walking as it is for anything else. For one thing, we carry our mind around with us 
when we walk, so we are usually absorbed in our own thoughts to one extent or another. We are hardly ever just walking, even when we are just going out for a walk. Walking meditation involves intentionally attending to the experience of walking itself.

\section{Vibration Meditation}

Also called sounding meditation, this technique uses the repetition of a word or sound as its focal point. Vibration meditation has appeal to those who find that making noise is a path to inner quiet. We are taught to be nice and quiet as little children. Releasing sound and noise helps us release stress. Get on your feet. Stand with your feet shoulder-width apart, your knees slightly bent and your hips centered, as though you are about to squat. Or, if you wish, sit or lie down. Keep your body loose and comfortable with your arms at your sides or on your hips. Begin by taking a few cleansing breaths. Pick a word, any word. Choose a word that alternates vowels and consonants-like "serenity." The word that you select does not necessarily have to be a spiritual one. It just has to feel good when you say it. Repeat after yourself. Repeat the word, chant the word, focus on nothing but saying the word repeatedly. Let the sound of the word vibrate through your body. Let the word resonate up from your abdomen and let it go to your hands, your feet. Let your muscles move as you chant the word. Some people have a tendency to clench their muscles when they are tense. It is important to roll the sound through your body so that you can clear out the tightness in your muscles. Doing so promotes the meditative state of relaxation that feels like a natural high.

\section{Mantra Meditation}

Prepare for your meditation as usual. A mantra is a word or a phrase that is repeated over and over as a means of achieving focus and concentration for deep meditation. The failsafe method of meditation is with mantras. No matter how much trouble you are having concentrating or getting time, a mantric meditation will always get you meditating quickly. It is also the easiest. 'Aum' is the most powerful mantra. It is able to reveal all the qualities of the soul. But choose a mantra you would like to use. Repeat it aloud or to yourself. Feel that the source of the mantra is in the deepest, inmost recesses of your heart and that you have to really focus, really concentrate to open the floodgates of that quality. Use the flow of your breath if you like to create the rhythm of the flow of energy.

\section{Body Scan Meditation}

Body Scan Meditation is often used by people who want to try a more formal type of mindfulness without attending a Yoga or Tai Chi class. Lie on your back with your legs uncrossed, your arms at your sides, palms up, and your eyes open or closed, as you wish. Focus on your Breathing, how the air moves in and out of your body. After several deep breaths, as you begin to feel comfortable and relaxed, direct your attention to the toes of your left foot. Tune into any sensations in that part of your body while remaining aware of your Breathing. It often helps to imagine each breath flowing to the spot where you're directing your attention. Focus on your left toes for one to two minutes. Then move your focus to the sole of your left foot and hold it there for a minute or two while continuing to pay attention to your breathing. Follow the same procedure as you move to your left ankle, calf, knees, thigh, hip and so on all around the body. Pay particular attention to any areas that cause pain or are the focus of any medical condition (for Asthma, the lungs; for Diabetes, the pancreas). Pay particular attention to the head: the jaw, chin, lips, tongue, roof of the mouth, nostrils, throat, cheeks, eyelids, eyes, eyebrows, forehead, temples and scalp.

Finally, focus on the very top of your hair, the uppermost part of your body. Then let go of the body altogether, and in your mind, hover above yourself as your breath reaches beyond you and touches the universe.

\section{Breath and Navel Meditation}

Breath and Navel Meditation is the oldest Meditation Method on record in China as well as India. It is also the method usually taught to 
Beginners. Breath and Navel Meditation works directly with the natural flow of breath in the nostrils and the expansion and contraction of the abdomen. This Taoist Meditation is a good way to develop focused attention and one-pointed awareness.

(1) Sit cross-legged on a cushion, on the floor, or upright on a low stool and adjust the body's posture until well balanced and comfortable. Press tongue to palate, close your mouth without clenching the teeth, and lower the eyelids until almost closed.

(2) Breath naturally through the nose, drawing the inhalation deep down into the abdomen and making the exhalation long and smooth. Focus your attention on two sensations, one above and the other below. Above, focus on the gentle breeze of air flowing in and out of the nostrils like a bellows. In exhalation, try to 'follow' the breath out as far as possible, from 3 to 18 inches. Below, focus on the navel rising and falling and the entire abdomen expanding and contracting like a balloon with each inhalation and exhalation. You may focus attention on the nostrils or the abdomen, or on both, or on one and then the other, whichever suits you best. From time to time, mentally check your Posture and adjust it if necessary. Whenever you catch your mind wandering off or getting cluttered with thoughts, consciously shift your attention back to your breath. Sometimes it helps to count either inhalations or exhalations, until your mind is stably focused.

\section{Central Channel Meditation}

Central Channel Meditation is an ancient Taoist method modified and taught by Master Han Yumo at his Sung Yang Tao Centers in Taiwan and Canada. It is a simple and effective way for Beginners to rapidly develop a tangible awareness of internal energy and a familiarity with the major power points through which energy is circulated and exchanged with the surrounding sources of heaven and earth. It relaxes the body, replenishes energy, and invigorates the spirit.

(1) Adopt a comfortable Sitting Posture. First, take a deep breath and bend forward slowly, exhaling audibly through the mouth in order to expel stale breath from the lungs; repeat three times. Then, sit still and breathe naturally, letting the abdomen expand and contract with each breath. However, instead of focusing attention on the flow of air through the nostrils, focus on the beam of energy entering the crown of the head at a point about two inches above the hairline, called the 'Medicine Palace'.

(2) Feel the beam of energy flowing in through this point as you begin each inhalation and follow it down through the Central Channel into the Lower Elixir Field below the navel, then follow it back up the Central Channel and out through the Medicine Palace point on exhalation. The sensation at the crown point is most noticeable at the beginning of inhalation and the end of exhalation and feels somewhat like a flap or valve opening and closing as energy flows through it. There may also be feelings of warmth, tingling, or numbness in the scalp, all of which are signs of energy moving under the scrutiny of awareness.

(3) After practicing this method for a while, your head may start to rock spontaneously back and forth or from side to side after fifteen or twenty minutes of sitting, or else your entire body may start trembling and shaking. This is a good sign, for it means that your channels are opening and that energy is coursing strongly through them. Try neither to suppress nor encourage these spontaneous tremors.

\section{Microcosmic Orbit Meditation}

This is the classic Taoist Meditation method for refining, raising, and circulating internal energy via the 'orbit' formed by the 'Governing Channel' from perineum up to head and the Conception Channel from head back down to perineum. Activating the Microcosmic Orbit is a key step that leads to more advanced practices. Taoists believe that Microcosmic Orbit Meditation fills the reservoirs of the Governing and Conception channels with energy, which is then distributed to all the major organ-energy meridians, thereby energizing the internal organs. It draws abundant energy up from the sacrum into the brain, thereby enhancing cerebral circulation of blood and stimulating secretions of vital neurochemicals. It is also the first stage for cultivating the 'spiritual 
embryo' or 'golden elixir' of immortality, a process that begins in the lower abdomen and culminates in the mid-brain. This is probably the best of all Taoist methods for cultivating health and longevity while also 'opening the three passes' to higher spiritual awareness.

(1) The first step is to still the body, calm the mind, and regulate the breath. With this settled mind, sit alone in a quiet room, senses shut and eyelids lowered. Turn your attention within, and inwardly visualize a pocket of energy in the umbilical region; within it is a point of golden light, clear and bright, immaculately pure. Focus attention on the navel until you feel the 'pocket of energy' glowing in the umbilical region. The breath through your nose will naturally become light and subtle, going out and in evenly and finely, continuously and quietly, gradually becoming slighter and subtler. When the feeling is stable and the energy there is full, use your mind to guide energy down to the perineum and back up through the aperture in the coccyx.

(2) Steadily visualize this true energy as being like a small snake gradually passing through the nine apertures of the coccyx. When you feel the energy has gone through this pass, visualize this true energy rising up to where the ribs meet the spine, then going through this pass and right on up to the Jade Pillow, the back of the brain.

(3) Then imagine your true spirit in the Nirvana Chamber in the center of the brain, taking in the energy. When this true energy goes through the Jade Pillow, press the tongue against the palate. The head should move forward and tilt slightly upwards to help it. When you feel this true energy penetrating the Nirvana Chamber, this may feel hot or swollen. This means the pass has been cleared and the energy has reached the Nirvana Center.

(4) Next, focus attention on the Celestial Eye between the eyebrows and draw energy forwards from the midbrain and out through the point between the brows. This may cause a tingling or throbbing sensation there. Then the center of the brows will throb - this means the Celestial Eye is about to open. Then move the spirit into the center of the brows and draw the true energy through the Celestial Eye. If you see the eighteen thousand pores and three hundred and sixty joints of the whole body explode open all at once, each joint parting three-tenths of an inch, this is evidence of the opening of the Celestial Eye. This is what is meant when it is said that when one pass opens all the passes open, and when one opening is cleared all the openings are cleared.

(5) You may wish to stay and work with this point for a few minutes, before letting energy sink down through the palate and tongue into the throat to the heart. This may feel as though there is cool water going down the Multistoried Tower of the windpipe. Do not swallow; let it go down by itself, bathing the bronchial tubes. Then the vital energy will bathe the internal organs and then return to the genitals. This is what is called return to the root.

(6) From the heart, draw it down through the Middle Elixir Field in the solar plexus, past the navel, and down into the Ocean of Energy reservoir in the Lower Elixir Field, where energy gathers, mixes, and is reserved for internal circulation. Then begin another cycle up through the coccyx to the mid-spine behind the heart and up past the Jade Pillow into the brain.

(7) Breathe naturally with your abdomen, and don't worry whether energy moves up or down on inhalation or exhalation; coordinate the flow of breath and energy in whatever manner suits you best. However, if you reach the stage where you can complete a full Microcosmic Orbit in a single breath, it's best to raise energy up from coccyx to head on exhalation and draw it down from Upper to Lower Elixir Field on inhalation. If you practice this way for a long time, eventually you can complete a whole cycle of ascent and descent in one visualization. If you can quietly practice this inner work continuously, whether walking, standing still, sitting, or lying down, then the vital energy will circulate within, and there will naturally be no problem of leakage. Chronic physical ailments, Taoists believe, will naturally disappear. This Meditation may also cause the head to rock or the body to tremble, which, Taoists believe, are signs of progress.

\section{Benefits of meditation}

Researches have shown that Meditation can contribute to an individual's psychological and physiological well-being. This is accomplished as 
Meditation brings the brainwave pattern into an alpha state, which is a level of consciousness that promotes the healing state. There is scientific evidence that Meditation can reduce blood pressure and relieve pain and stress. Benefits of meditation can be divided into three parts: (1) physiological benefits; (2) psychological benefits; and (3) spiritual benefits.

\section{Physiological Benefits}

(a) Deep rest-as measured by decreased metabolic rate, lower heart rate, and reduced workload of the heart. (b) Lowered levels of cortisol and lactate-two chemicals associated with stress. (c) Reduction of free radicals- unstable oxygen molecules that can cause tissue damage. (d) Decreased high blood pressure. (e) Higher skin resistance. Low skin resistance is correlated with higher stress and anxiety levels. (f) Drop in cholesterol levels. High cholesterol is associated with cardiovascular disease. (g) Improved flow of air to the lungs resulting in easier breathing. This has been very helpful to asthma patients. (h) Decreases the aging process. (i) Higher levels of DHEAS in the elderly, an additional sign of youthfulness.

\section{Psychological Benefits}

(a) Increased brain wave coherence Greater creativity (c) Decreased anxiety

(d) Decreased depression (e) Decreased irritability and moodiness (f) Improved learning ability and memory (g) Increased self-actualization. (h) Increased feelings of vitality and rejuvenation (i) Increased happiness (j) Increased emotional stability.

\section{Spiritual Benefits}

The longer an individual practices meditation, the greater the likelihood that his or her goals and efforts will shift toward personal and spiritual growth. Many individuals who initially learn meditation for its self-regulatory aspects find that as their practice deepens they are drawn more and more into the realm of the "spiritual." In her work with many cancer and AIDS patients, Dr. Borysenko has observed that many are most interested in meditation as a way of becoming more attuned to the spiritual dimension of life. She reports that many die "healed," in a state of compassionate self-awareness and selfacceptance.

\section{References}

1. Shapiro, D.H. (1982). Overview: Clinical and physiological comparison of meditation with other self-control strategies. American Journal of Psychiatry, 139, 267-274.

2. Seeman, W., Nidich, S., \& Banta, T. (1972) Influence of transcendental meditation on a measure of self-actualization. Journal of Counseling Psychology, 19, 184 - 187.

3. Goenka, S.N. (2001). Discourses on SatipatthanaSutta. Igatpuri: Vipassana Research Institute.

4. Brownstein, A.H., \&Dembert, M.L. (1989). Treatment of essential hypertension with yoga relaxation therapy in a USAF aviator: A case report. Aviation, space, \& environmental medicine, 60, $684-687$.

5. Beary, J.F., \& Benson, H.A. (1974). A simple physiologic technique which elicits the hypometabolic changes of the relaxation response. Psychosomatic Medicine, 36, 115 120.

6. Carrington, P. (1977). Freedom in meditation. Garden City. NY: Doublebay.

\begin{tabular}{|l|l|}
\hline \multicolumn{2}{|c|}{ Access this Article in Online } \\
\hline & Website: \\
\hline & www.ijcrims.com \\
\hline Quick Response Code & Subject: \\
\end{tabular}

How to cite this article:

Abdul Wahab Pathath (2017). Meditation: Techniques and Benefits. Int. J. Curr. Res. Med. Sci. 3(6): 162-168. DOI: http://dx.doi.org/10.22192/ijcrms.2017.03.06.021 